\title{
Body Navigation-loaded Ultrasound Acquisition Technology: a Pilot Comparison With Conventional Ultrasound
}

\section{Ki Choon Sim}

Korea University Anam Hospital

Beom Jin Park ( $\nabla$ radiolbj226@gmail.com )

College of Medicine, Korea University, Anam Hospital https://orcid.org/0000-0002-7289-3683

Byunjun Kim

Korea University Anam Hospital

\section{Yeo Eun Han}

Korea University Anam Hospital

\section{$\mathrm{Na}$ Yeon Han}

Korea University Anam Hospital

\section{Min Ju Kim}

Korea University Anam Hospital

\section{Deuk Jae Sung}

Korea University Anam Hospital

\section{Sang Hyun Park}

Korea University Anam Hospital

\section{Kwang-Sig Lee}

Korea University Anam Hospital

\section{Yongwon Cho}

Korea University Anam Hospital

\section{Research article}

Keywords: Ultrasound, annotation, body mark, navigation, transducers

Posted Date: August 6th, 2021

DOl: https://doi.org/10.21203/rs.3.rs-781540/v1

License: (c) (1) This work is licensed under a Creative Commons Attribution 4.0 International License.

Read Full License 


\section{Abstract}

Background: To investigate the usefulness of body navigation-loaded ultrasound including a real time transducer location and the inspection site compared with conventional ultrasound images.

Methods: Under the approval of institutional review board, we prospectively enrolled total 29 healthy adult volunteers. One gastrointestinal radiologist performed abdominal ultrasound simultaneously using Ultrasound Navigation Image Convergence System developed by researchers. Subsequently, an equivalent conventional ultrasound image set was prepared. Three radiologists independently evaluated the two ultrasound image sets regarding the recognition of the target organ (2-points), the transducer location (2-points), and the transducer orientation (1-point). At intervals of two-weeks, conventional ultrasound images were analyzed first, and body navigation-loaded images were later analyzed. The score differences between the first and second evaluations were compared using the Wilcoxon signed rank test. Inter-rater agreement of three reviewers was obtained by the Fleiss' Kappa test.

Results: A total of 1402 navigation-loaded ultrasound images were obtained. Ultrasound operator carefully selected a total of 203 images for analysis. In all three reviewers, the interpretation score of each evaluation was significantly increased in the second analysis using the body navigation-loaded ultrasound image (in reviewer $A$, from $4.07 \pm 1.56$ to $4.79 \pm 0.69$ points; in reviewer $B$, from $3.83 \pm 1.59$ to $4.49 \pm 0.88$ points; in reviewer $C$, from $3.43 \pm 1.60$ to $4.19 \pm 1.01$ points; $P<.0001$ ). The inter-rater agreement of each evaluation also increased significantly in the second analysis using the body navigation-loaded ultrasound image $(P<.0001)$.

Conclusion: The body navigation-loaded ultrasound imaging system allows other medical staffs to easily and accurately interpret ultrasound images.

\section{Background}

Ultrasound is an economical, non-invasive, and effective imaging modality used in almost all clinical practice. In conventional ultrasound, the ultrasound image is not automatically labeled with information regarding the location or orientation of the transducer. There is also no information about the part of the body being examined. Although variedly implemented in different countries, ultrasound images without body marks may be interpreted from the image itself, the operator's memory, and so on, and can therefore lose their value as an objective image. In clinical practice, knowledge of the transducer location and orientation may not be necessary because standardized views are widely obtained using ultrasound [1]; these have been optimized for their utility in differentiating normal from abnormal findings. However, accurate information about the transducer location or orientation could be important in ultrasound scans where the standardized view is less established or information regarding the transducer and patient's posture change may be required.

To overcome these problems, annotation using a body mark or text is essential. However, there is a disadvantage in that the operator must expend additional time and effort using various control buttons. 
Moreover, the ultrasound examination site intended by the operator and the body mark may not exactly match, and there may be errors during the manipulation process. To complement the existing body mark system of ultrasound, several methods have been developed to install equipment that enables threedimensional (3D) position calculation, on the ceiling of the ultrasound room or the probe itself, and a tracking system that can monitor the position of a device within a subject and superimpose a graphic symbol on the diagnostic image of the subject [2-4]. However, these methods also require the transducer to have additional hardware, such as a gyro sensor and an acceleration sensor, and these methods seem to be difficult to apply in clinical practice.

We devised a novel technology that minimizes the necessity for complex hardware and intuitively includes ultrasound location information in a conventional ultrasound image. This technology was designed to be applicable to various organs and to be capable of recording the transducer position with respect to the body. It used a 3D depth camera capable of photographing the inspection site with the transducer and a software specially designed to merge the acquired image with the conventional ultrasound image. The technology was experimentally applied to the most frequently performed abdominal ultrasound, and this pilot study aimed to investigate the accuracy of image interpretation and the effectiveness of body navigation-loaded ultrasound, which included a real-time transducer location and an inspection site, compared with conventional ultrasound.

\section{Materials And Methods Study population}

With the approval of the institutional review board, we prospectively enrolled 29 healthy adult participants through in-hospital recruitment. Informed consent was obtained from all participants. In addition, consent was obtained to publish the body navigation-loaded ultrasound images obtained in this study. From December 2018 to January 2019, one gastrointestinal radiologist performed abdominal ultrasound of various organs in these participants, with or without NPO (nothing by mouth) for the participants' convenience. In two participants, with additional informed consent, ultrasound was applied to areas other than the abdomen to assess the applicability of this technology in various organs.

\section{Concept of body navigation-loaded ultrasound acquisition technology}

This technology uses a commercial 3D depth camera positioned to capture the ultrasound scene within the vicinity of the patient at the same time when the operator obtains a conventional ultrasound image. The images taken with the inspection site and transducer location are combined in real time in the form of thumbnails and inserted into conventional ultrasound images using a commercial computer and gateway system [5] containing a 3D mesh filter [6], which are then automatically transmitted to the picture archiving and communication system (PACS) server (Fig. 1). 


\section{Ultrasound protocol and acquisition of navigation-loaded images}

Ultrasound was performed by a radiologist with 8 years of experience in gastrointestinal radiology using an iU22 ultrasound machine (Philips, Seattle, WA, USA) with a $5 \mathrm{MHz}$ convex transducer. In the ultrasound image, additional information, such as graphic body marks or text, was not attached. Instead, the transducer and inspection site were captured using a Kinect v2 camera (Microsoft Corporation, Redmond, WA, USA), which can provide 3D depth information and object recognition using a software development kit. The camera was connected to the arm behind the ultrasound monitor using an overhead camera stand and fixed such that the inspection site could be viewed from above. In addition, we used a specialized software called MeshGateway, developed by our researchers, which included the Ultrasound Navigation Image Convergence System using 3D Depth Measurement Technique and the ability to transmit navigation-loaded images to the PACS server (Fig. 2). The process of installing the camera and preparing the software took approximately 1 to 2 minutes. If the inspection site was the same and there was no need to manipulate the camera and software, no additional time would be required thereafter.

\section{Preparation for image analysis}

A total of 1,402 body navigation-loaded images were acquired from 29 participants. The ultrasound operator carefully selected seven images per patient. Inappropriate images, such as those captured when the transducer was moving or those that were out of focus were excluded. As a result, 203 images were selected for analysis, which formed the body navigation-loaded ultrasound image set. Subsequently, an equivalent conventional ultrasound image set was prepared. Two image sets of identical organs were created as portable document format files for image analysis.

\section{Ultrasound image analysis and scoring}

Two board-certified gastrointestinal radiologists (Reviewer A with 20 years of experience and Reviewer B with 10 years of experience, respectively) and one trainee radiologist (Reviewer $C$ with 2 years of experience) independently evaluated the two image sets. The conventional ultrasound image set was evaluated first, followed by the body navigation-loaded ultrasound image set, two weeks later.

The reviewers were instructed to assess ultrasound images based on the following: 1) recognition of the target organs (2-points) such as specific liver segments, the extrahepatic bile duct, gallbladder, pancreas, spleen, kidneys, stomach, small and large bowels, urinary bladder, abdominal muscles (e.g., psoas muscle), aorta, or the uterus; 2 ) estimation of transducer location according to the nine regions of the abdomen (2-points) [7]; and 3) estimation of transducer orientation (1-point): transverse, longitudinal, or oblique orientations.

The target organ and transducer location and orientation intended by the ultrasound operator were considered gold standards, and each ultrasound image was scored from a maximum of 5 points to a minimum of 0 (Fig. 3). 
For recognition of the target organ, when the target organ was correctly recognized and the directions (right or left) and exact location were accurately specified, a score of two points was assigned. If the direction was incorrect or the exact anatomical structure was not specified, one point was assigned. If it was perceived as a different organ, a score of 0 was assigned.

For the scoring of the transducer location, if it matched the ultrasound operator's answer, a score of two points was assigned, and if the location was evaluated as immediately adjacent in nine regions [7], one point was assigned.

The transducer orientation scoring was assigned one point if the orientation matched the ultrasound operator's answer. Because the transducer angle could be changed freely during the examination, an angle variation of the transducer within approximately $10^{\circ}$ was defined as a transverse or longitudinal orientation.

\section{Statistical analysis}

The mean difference between the evaluations was obtained by the Wilcoxon signed-rank test using IBM SPSS Statistics software, version 20.0, for Windows (IBM Corp., Armonk, NY, USA). Inter-rater agreement of three reviewers was obtained by the Fleiss' Kappa test using SAS (version 9.4; SAS Institute Inc., Cary, NC, USA). Statistical significance was set at $P<0.05$.

\section{Results}

Twenty-two men and seven women were recruited for this study (mean age: 45.1 years, range: 27-59 years). Images of various organs of the abdomen were taken, and standard images were taken for the liver, pancreas, kidneys, and urinary bladder (Fig. 4).

The 203 selected ultrasound images were classified according to the organs as follows: liver (including portal and hepatic veins, $n=59)$, extrahepatic bile duct $(n=12)$, gallbladder $(n=5)$, pancreas $(n=19)$, spleen $(n=15)$, kidneys $(n=43)$, urinary bladder $(n=16)$, bowel $(n=6)$, psoas muscle $(n=20)$, aorta $(n=$ $5)$, uterus $(n=2)$, and vertebra $(n=1)$.

For reviewer $A$, the interpretation scores were $4.10 \pm 1.50$ points for the first analysis using the conventional ultrasound image set and $4.76 \pm 0.63$ points for the second analysis using the body navigation-loaded ultrasound image set. For reviewer $B$, the first and second analysis scores were $3.82 \pm$ 1.53 and $4.40 \pm 0.90$ points, respectively. For reviewer $C$, the first and second analysis scores were 3.43 and 4.19 points, respectively. There was a statistically significant increase in the interpretation scores of all reviewers $(P<0.001)$ (Table 1$)$. 
Table 1

Average score difference between the first and second analysis

\begin{tabular}{|llll|}
\hline & First analysis & Second analysis & $P$ value \\
\hline Reviewer A & $4.10 \pm 1.50$ & $4.76 \pm 0.63$ & $<0.001$ \\
\hline Reviewer B & $3.82 \pm 1.53$ & $4.40 \pm 0.90$ & $<0.001$ \\
\hline Reviewer C & $3.43 \pm 1.60$ & $4.19 \pm 1.01$ & $<0.001$ \\
\hline \multicolumn{4}{|l}{ Data are expressed as mean \pm standard deviation. } \\
\hline
\end{tabular}

Among the three reviewers, the mean score difference in each analysis session was statistically different in both the conventional ultrasound image set and the body navigation-loaded ultrasound image set (Table 2).

Table 2

Average score difference among the three reviewers in each analysis

\begin{tabular}{|lllll|}
\hline Reviewer & First analysis & $P$ value & Second analysis & $P$ value \\
\hline A vs B & $4.10 \pm 1.50$ vs & 0.006 & $4.76 \pm 0.63$ vs & $<0.001$ \\
& $3.82 \pm 1.53$ & & $4.40 \pm 0.90$ & \\
B vs C & $3.82 \pm 1.53$ vs & 0.002 & $4.40 \pm 0.90$ vs & $<0.001$ \\
& $3.43 \pm 1.60$ & & $4.19 \pm 1.01$ & \\
A vs C & $4.10 \pm 1.50$ vs & $<0.001$ & $4.76 \pm 0.63$ vs & $<0.001$ \\
& $3.43 \pm 1.60$ & & $4.19 \pm 1.01$ & \\
\hline Data are expressed as mean \pm standard deviation. & \\
\hline
\end{tabular}

In the first analysis, the inter-rater agreement of target organ recognition showed moderate agreement (Fleiss' Kappa value of 0.610 ), and the estimation of the transducer location and transducer orientation showed weak agreement (Fleiss' Kappa values of 0.425 and 0.571 , respectively). In the second analysis, the kappa value in all evaluation categories increased and showed a moderate agreement (Fleiss' Kappa value from 0.619 to 0.792 ). In both the first and second evaluations, the agreement for the transducer location was the lowest (Table 3). 
Table 3

Inter-rater agreement of three reviewers in each analysis using Fleiss' Kappa test
Rating category $(n=203)$
Fleiss' Kappa value $(95 \% \mathrm{Cl})$
$P$ value

First analysis

Target organ recognition

$0.610(0.551-0.669)$

$<0.001$

Probe location

$0.425(0.364-0.485)$

$<0.001$

Probe orientation

$0.571(0.500-0.642)$

$<0.001$

Second analysis

Target organ recognition

$0.792(0.743-0.841)$

$<0.001$

Probe location

0.619 (0.552-0.685)

$<0.001$

Probe orientation

$0.760(0.702-0.819)$

$<0.001$

The Fleiss' Kappa value result was interpreted as follows: $0-0.20$, none; $0.21-0.39$, minimal; $0.40-$ 0.59 , weak; $0.60-0.79$, moderate; $0.80-0.90$, strong; and above 0.90 , almost perfect. $\mathrm{Cl}$, confidence interval.

\section{Discussion}

In this pilot study, the authors acquired body navigation-loaded ultrasound images that included information regarding the inspection site and transducer location. In the ultrasound image analysis, the mean score of interpretations of body navigation-loaded ultrasound images increased significantly for all raters compared with conventional ultrasound images without body marker. In addition, it was confirmed that the inter-rater agreement was improved in the second analysis that used the navigation-loaded ultrasound image set. These results were obtained not by physicians but by experienced gastrointestinal radiologists who are familiar with ultrasound imaging. Thus, body navigation-loaded ultrasound imaging is expected allow radiologists to interpret ultrasound images more accurately and objectively. The authors also believe that it will certainly be helpful to physicians who may be unfamiliar with the ultrasound image. However, based on our results, the presence or absence of navigation-loaded images did not significantly affect the target organ recognition of easily recognizable organs, such as the left lateral section of the liver, portal vein bifurcation, spleen, or urinary bladder. These organs were recognized nearly $100 \%$ of the time in all the primary and secondary analyses.

In the present study, there was a statistically significant difference in the interpretation scores of the reviewers (Table 2). Reviewer A (senior radiologist) had a higher interpretation score. Apart from the low scores of the less experienced resident, the difference in the scores of the secondary evaluation in which the two experienced radiologists interpreted the navigation-loaded ultrasound image was caused by the transducer location interpretation. When evaluating the boundaries of nine regions of the abdomen, the senior radiologist's interpretation of the transducer location was more consistent with the operator's intention. Because subjective interpretation of the boundary area was possible, the inter-rater agreement 
of the transducer location also showed lower agreement than that of the other evaluation categories (Table 3). If the four abdominal quadrants with a clear anatomical landmark of the umbilicus were used as the standard for transducer location interpretation, it was expected that all raters would show a higher interpretation score and a high degree of agreement.

The most important advantage of navigation-loaded ultrasound is that it increases the operator's convenience and is expected to allow the operator to focus solely on ultrasound examination. In some cases, the operator's increased effort may be required while adding appropriate body marks or text available on the ultrasound equipment to ultrasound images. This process is cumbersome and timeconsuming because radiologists or clinicians must make a diagnosis while taking ultrasound images and add a body mark to the images using various control buttons. Recently, using commercially available products like touch screen labels and scan assistant make the process of labeling very stream-lined and easy [8]. However, the authors' navigation-loaded image can minimize this process because the exact information regarding the transducer and inspection site is automatically integrated with the ultrasound image in real time. Thus, this navigation-loaded ultrasound image is expected to assist in interpreting ultrasound images where the marking of specific locations is required, or where the distinction between the right and left sides is important. The disadvantage of the authors' semi-automated technology compared to the existing ultrasound body mark system is that it may take a short time to fix the camera and set the software just before starting the ultrasound examination.

The body navigation-loaded ultrasound technology developed by us did not require highly advanced skills or equipment, but there were some issues that had to be addressed during its development. The first issue was to protect patients' privacy, such as faces or breasts. This study aimed to minimize the exposure of sensitive areas of the body by applying a 3D mesh filter while maintaining the shape, size, and ratio of the body taken by the 3D depth camera. A 3D depth camera allows utilize an object recognition function that cannot be implemented with a general camera, thereby the body can be animated. Additionally, the location of the transducer could appear as it was, without modification. The result was a more intuitive understanding of the navigation-loaded ultrasound image. The second issue was to determine how to simultaneously acquire ultrasound images and 3D depth images to increase operator convenience. Because it is not efficient to use the ultrasound image acquisition button and the camera capture button separately, this problem was solved by setting the camera to simultaneously capture an image when the ultrasound image acquisition button was pressed, using the MeshGateway software on a computer connected to the ultrasound system. The third issue was to determine how much inspection site and transducer information should be included in the ultrasound image in photos taken with a 3D depth camera. With the camera fixed in a position above the patient's head, body parts other than the inspection site were cropped, and adjustments were required before starting the ultrasound examination so that the inspection site and location of the transducer did not deviate from the cropped area. After this issue was resolved, it was necessary to decide where to place a navigation-loaded image on the conventional ultrasound image. Care was taken not to overlap the information regarding the ultrasound parameters or scanned ultrasound images. Depending on the ultrasound vendor and specific model, it was expected to be variable, but we considered it better to insert the thumbnail in the upper right part of the ultrasound 
image. While using convex transducers, navigation-loaded image could be properly inserted into the empty portion of the ultrasound image that appeared to be radial.

There are several limitations to this pilot study using this experimental technology. First, the diagnostic usefulness of this technology compared to the conventional ultrasound body mark system has not been evaluated. Comparing different methods of labeling images may have resulted in better outcome. Some physicians may feel that the technology has no significant advantage over the current graphic body mark system. Although this pilot study was conducted only on abdominal ultrasound, where the standard view protocol is widely used, it is expected to be useful in other organs such as the musculoskeletal joints, peripheral vascular systems, and breast, where the inspection site and transducer information is more important than in the abdomen. Figure 5 demonstrates that this technology appears to be useful for identifying ultrasound images of various organs. Second, protecting patients' privacy and increasing the accuracy of the inspection site and transducer information are trade-offs for each other. In this study, navigation-loaded images were acquired with a low-resolution thumbnail, focusing on the protection of the participants' privacy. In addition, we are developing a program using artificial intelligence that automatically crops sensitive areas [9, 10], removes patients' faces [9-11], or completely replaces them with a simple body mark according to the inspection site and maintains the appropriate field of view. Nevertheless, due to patients' privacy issues, it is expected to be of little or limited use in gynecologic transvaginal or translabial ultrasound. The solution to this is to turn off the camera function in the software and then proceed with the conventional ultrasound examination. This method can be easily applied even if the patient refuses a new body marking system. Third, because camera installation and special software settings are required before starting the ultrasound scan, this process would be quite cumbersome. To overcome these shortcomings, the authors plan to develop an embedded system that applies the aforementioned artificial intelligence technology. Nevertheless, the authors' method is still applicable only to a permanent ultrasound room, and it will be difficult to apply to a portable ultrasound system. Fourth, our system is expected to be inaccurate for cineloop images because it only provides information about the static ultrasound transducer. However, we will be able to express the movement of the probe if necessary through software improvements in the future. Fifth, although the inter-rater agreement in the body navigation-loaded image increased, the kappa value did not increase almost perfectly. Thus, it cannot completely replace existing annotation methods such as text or arrows in a specific condition.

\section{Conclusions}

According to this pilot study, body navigation-loaded ultrasound technology is expected to assist in interpreting ultrasound images of suitable organs easily and objectively, and it is expected to improve the operator's convenience by reducing the use of body marks or text mark systems. In addition, the authors are expected to be able to apply this technology to various body organs through further improvements in the technology. 


\section{Abbreviations}

3D: three-dimensional; NPO: nothing by mouth; PACS: picture archiving and communication system.

\section{Declarations}

\section{Ethic approval and consent to participate}

Under the approval of the institutional review board of Korea University Anam Hospital (IRB No. 2018AN0424), we prospectively enrolled 29 healthy adult participants through in-hospital recruitment. Informed written consent was obtained from all participants.

\section{Consent for publication}

All authors agree with publication of this manuscript.

\section{Availability of data and materials}

The datasets used or analyzed during the current study available from the corresponding author on reasonable request.

\section{Competing interests}

The authors declare that they have no conflicts of interest.

\section{Funding}

This research was supported by a grant from Korea University Anam Hospital, Seoul, Republic of Korea (Grant No. K1809771 and No. 02000221) and a grant from Korea Technology and Information Promotion Agency for SMEs (Grant No. S2452341).

\section{Authors' contributions}

KCS and BJP proposed methods, performed the computations, and wrote the paper. BJP devised and supervised the project. BJP guaranteed the integrity of the entire study. BJK, YEH, MJK, and DJS contributed to interpretation of data. BJP and NYH contributed to analyze and interpret data. SHP, KSL, and YWC solved some detailed problems in the experiment of artificial intelligence. All authors read and approved the final manuscript.

\section{Acknowledgements}

None

\section{References}


1. Choi BI. Ultrasound diagnosis of the abdomen. Seoul. Ilchokak, 2015. pp. 102-123.

2. Hazas M, Ward A. . A Novel Broadband Ultrasonic Location System. International Conference on Ubiquitous Computing. 2002;2498:264-280.

3. Khaw KL, Sridharan A, Poznick L, Kilbaugh TJ, Hwang M. Probe position sensor to track image location in 2D ultrasound. Ultrasonics. 2020; 103:106084.

4. Darrow RD, Dumoulin CL, Souza SP. Imaging of interventional devices during medical procedures. In.: General Electric Co, 1997.

5. Hsiao CH, Kao T, Fang YH, Wang JK, Guo WY, Chao LH, Yen SH. System integration and DICOM image creation for PET-MR fusion. J Digit Imaging 2005;18:28-36.

6. Maglo A, Courbet C, Alliez P, Hudelot C. Progressive compression of manifold polygon meshes. Comput Graph 2012;36:349-359.

7. Cunningham DJ. Delimitation of regions of abdomen. J Anat Physiol. 1893;27(Pt 2):257-277.

8. GE LOGIC Club - Scan Assistant. https://www.logiqclub.net/korea/generalnews?id=522. Accessed 6 July 2021.

9. Redmon J, Farhadi A. YOLO9000: Better, Faster, Stronger. 2016; arXiv:1612.08242.

10. Lui W, Anguelov D, Erhan D, Szegedy C, Reed S, Fu CY, Berg AC. SSD: Single Shot MultiBox Detector. 2015; arXiv:1512.02325

11. Zhu JY, Park TS, Isola P, Efros AA. Unpaired Image-to-Image Translation using Cycle-Consistent Adversarial Networks. In: Proceedings of the IEEE International Conference on Computer Vision. 2017;pp2242-2251.

\section{Figures}

\section{Ultrasound equipment}

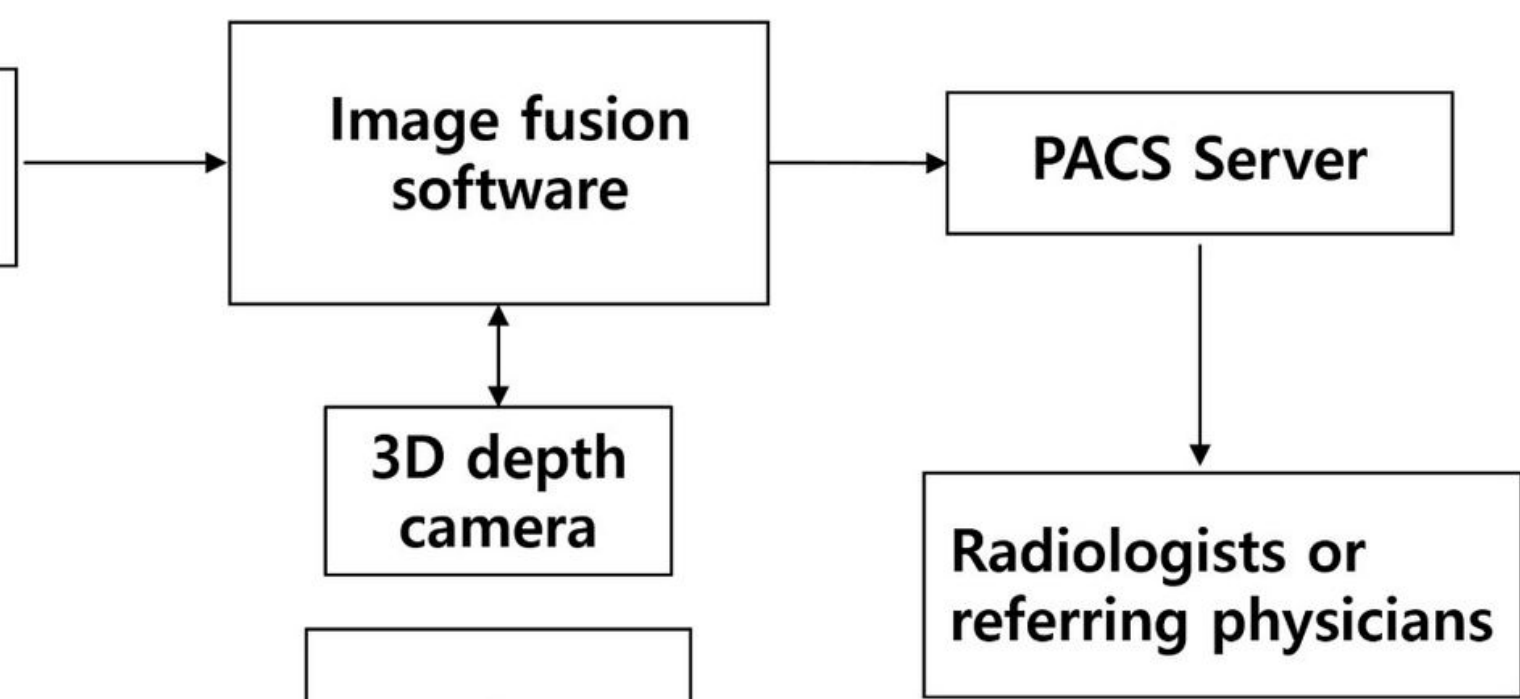

Patient 


\section{Figure 1}

Flow diagram showing image fusion workflow. 3D, three-dimensional; PACS, picture archiving and communication system.

a

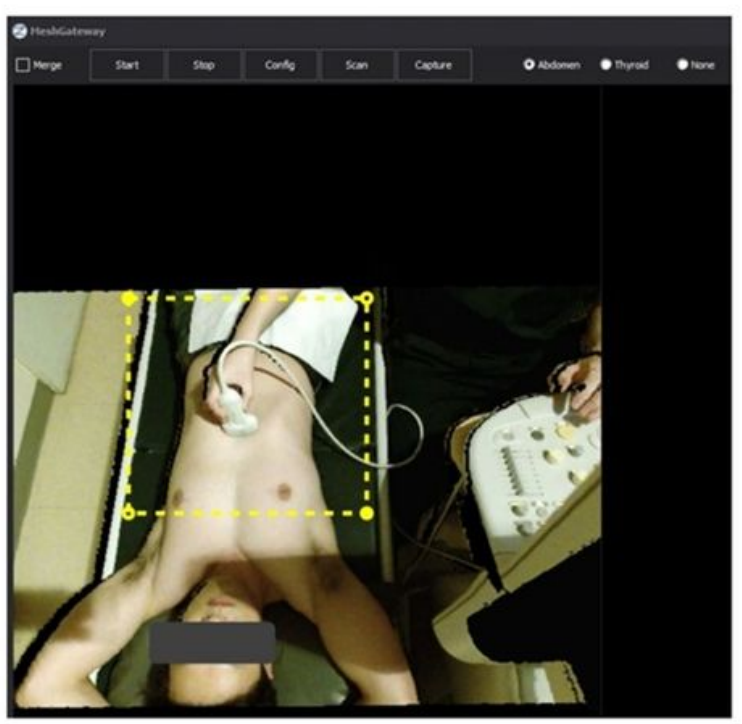

$\mathrm{b}$

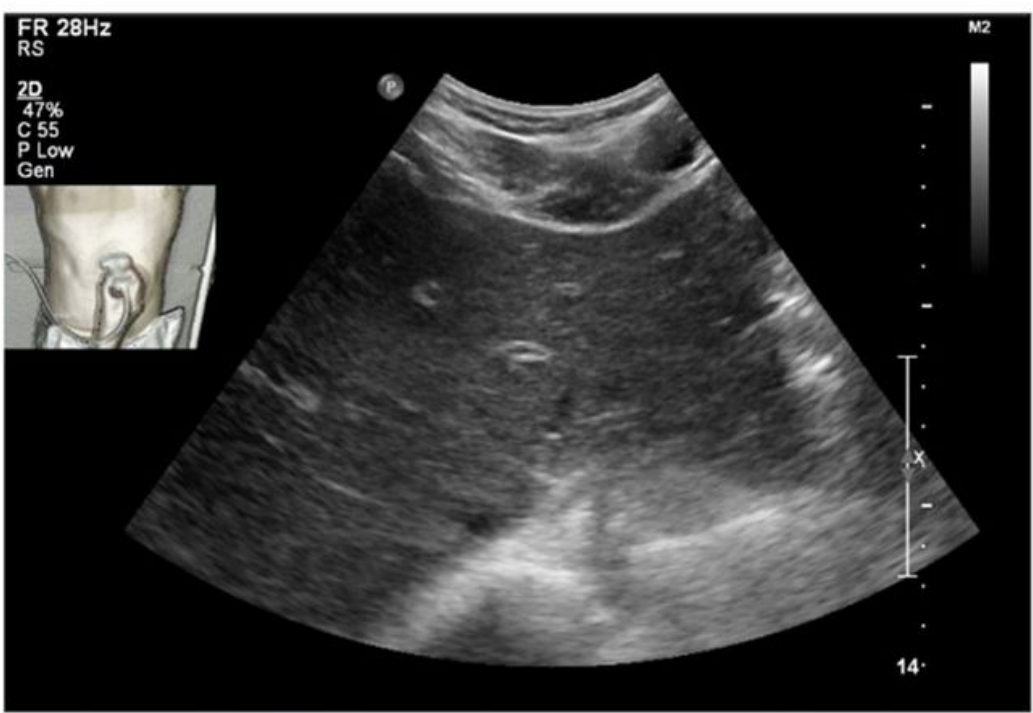

\section{Figure 2}

Ultrasound navigation convergence system (MeshGateway) work screen and navigation-loaded ultrasound image. (a) The image taken by the three-dimensional depth camera installed on the participant's head is transmitted to MeshGateway and appears on the work screen. Only the yellow dashed box is used for image fusion, and it can be adjusted according to the patient's position or inspection site. (b) A navigation-loaded ultrasound image taken by a transverse scan of the participant's left hemi-liver. The top left of the image shows a thumbnail containing information regarding the inspection site and transducer. 


\section{a}

1st interpretation with conventional ultrasound image set
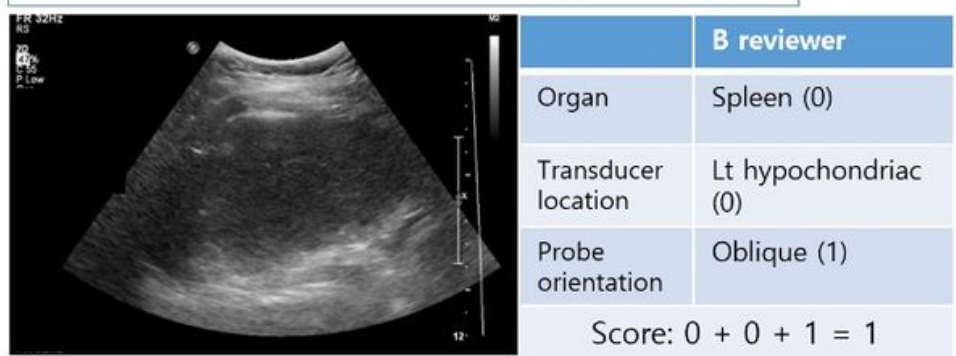

b

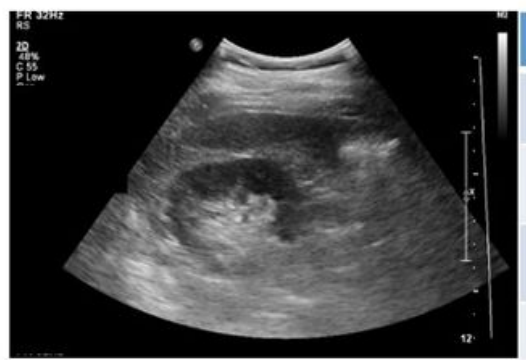

\section{2nd interpretation with}

body navigation-loaded ultrasound image set

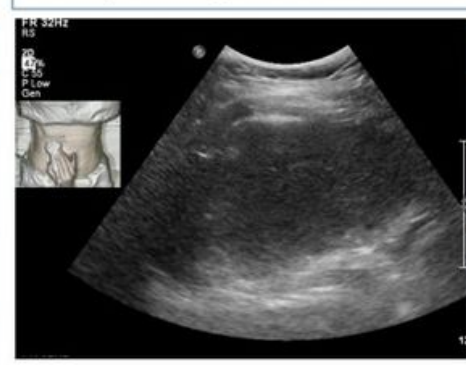

\begin{tabular}{|l|l|}
\hline \multicolumn{1}{|l|}{ Organ } & Liver dome (2) \\
\hline $\begin{array}{l}\text { Transducer } \\
\text { location }\end{array}$ & Epigastric (2) \\
\hline $\begin{array}{l}\text { Probe } \\
\text { orientation }\end{array}$ & Oblique (1) \\
\hline \multicolumn{1}{|c|}{ Score: $2+2+1=5$} \\
\hline
\end{tabular}

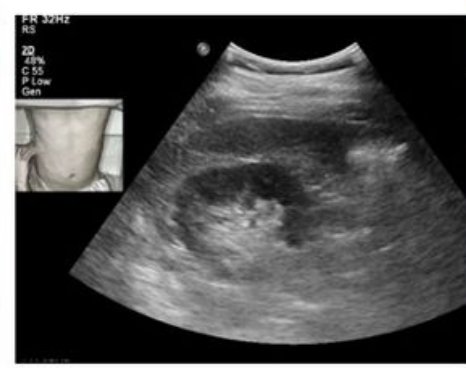

\begin{tabular}{|l|l|}
\hline & B reviewer \\
\hline Organ & Rt kidney (2) \\
\hline $\begin{array}{l}\text { Transducer } \\
\text { location }\end{array}$ & Rt lumbar (2) \\
\hline $\begin{array}{l}\text { Probe } \\
\text { orientation }\end{array}$ & Transverse (1) \\
\hline \multicolumn{1}{|c|}{ Score: $2+2+1=5$} \\
\hline
\end{tabular}

\section{Figure 3}

Ultrasound image interpretation and scoring. (a) This case was evaluated as an oblique scan of the left hypochondriac region by identifying the ultrasound image to be of a spleen in the first interpretation. However, in the second interpretation, it was confirmed that the transducer location was an epigastric region, and thus, reviewer $B$ could accurately identify that the liver dome was captured by ultrasound. (b) In the first interpretation session of reviewer B, the transverse scan of the kidney can be perceived; however, the left and right kidneys cannot be distinguished. Therefore, the first interpretation scored 2 points. However, the second interpretation using body-navigation-loaded ultrasound images shows that it is a transverse scan of the right kidney. 


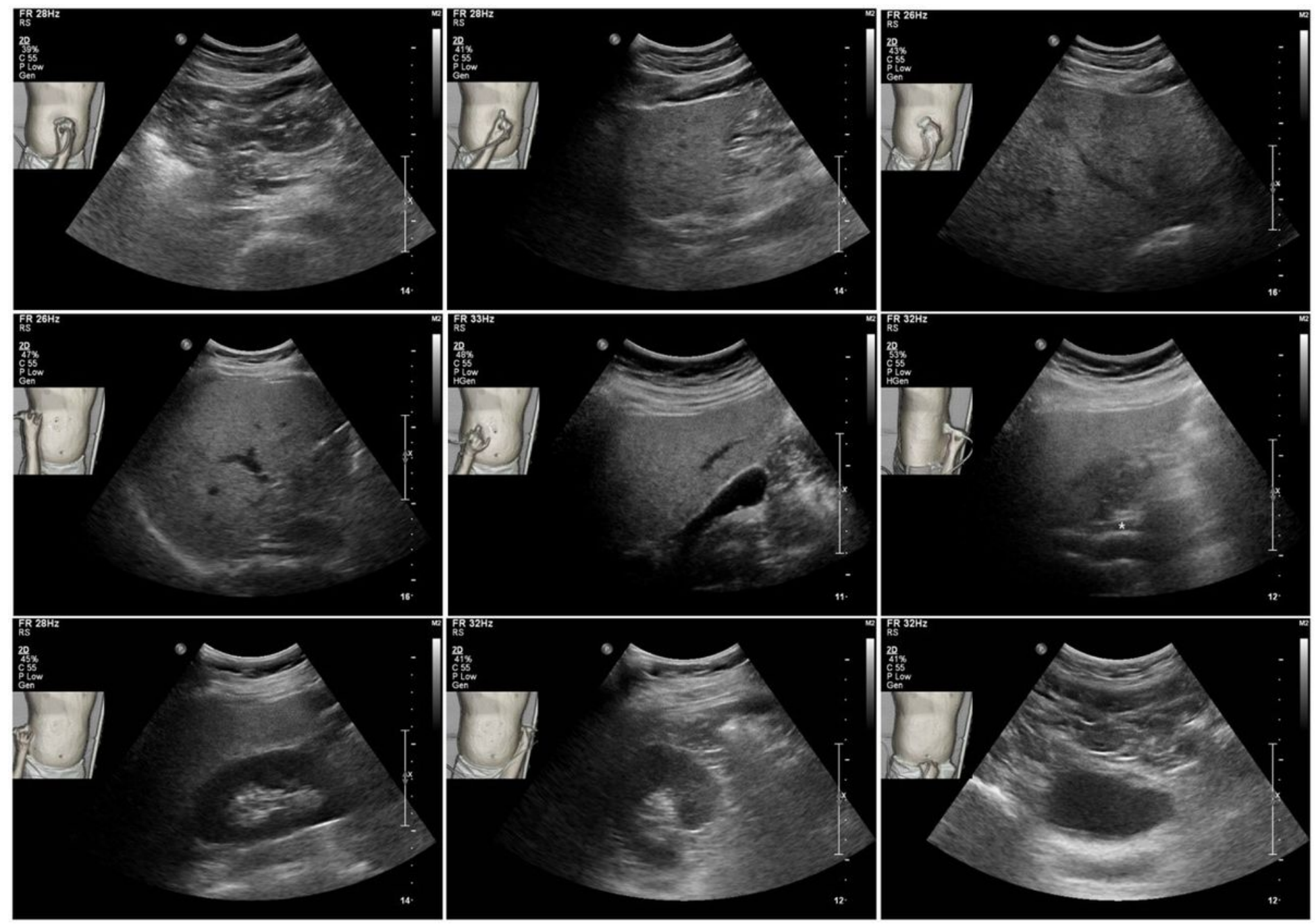

Figure 4

Application of body navigation-loaded ultrasound in the abdomen of one of the participants. The pancreatic body, several hepatic regions, gallbladder, extrahepatic bile duct, right kidney, left kidney, and urinary bladder are shown in order. The last ultrasound in the middle row is an image of the extrahepatic bile duct (asterisk), indicating that it has been taken in the left lateral decubitus position. 
a

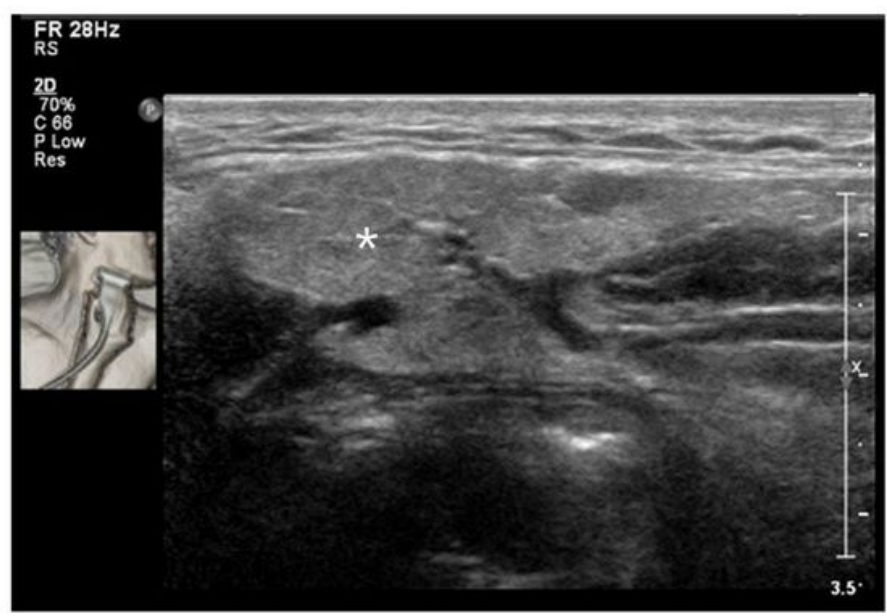

C

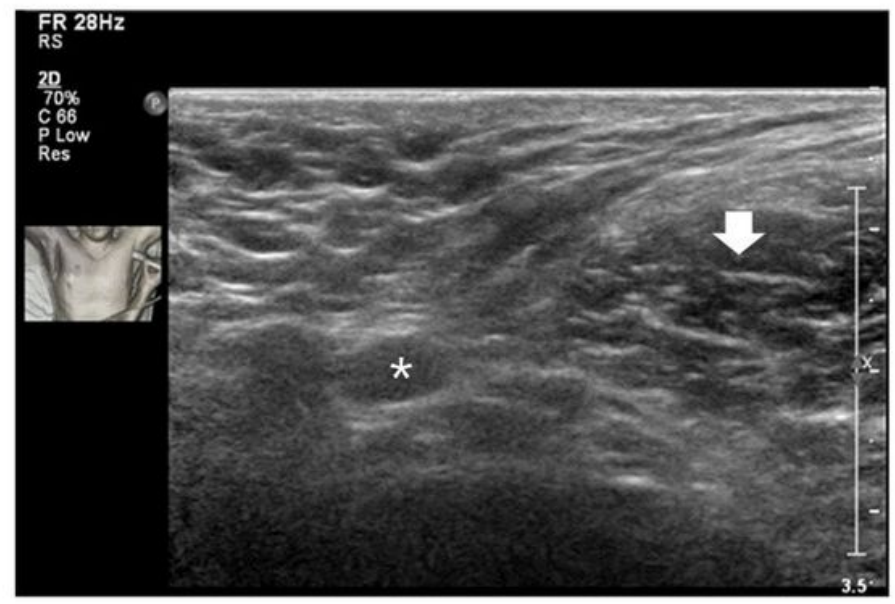

b
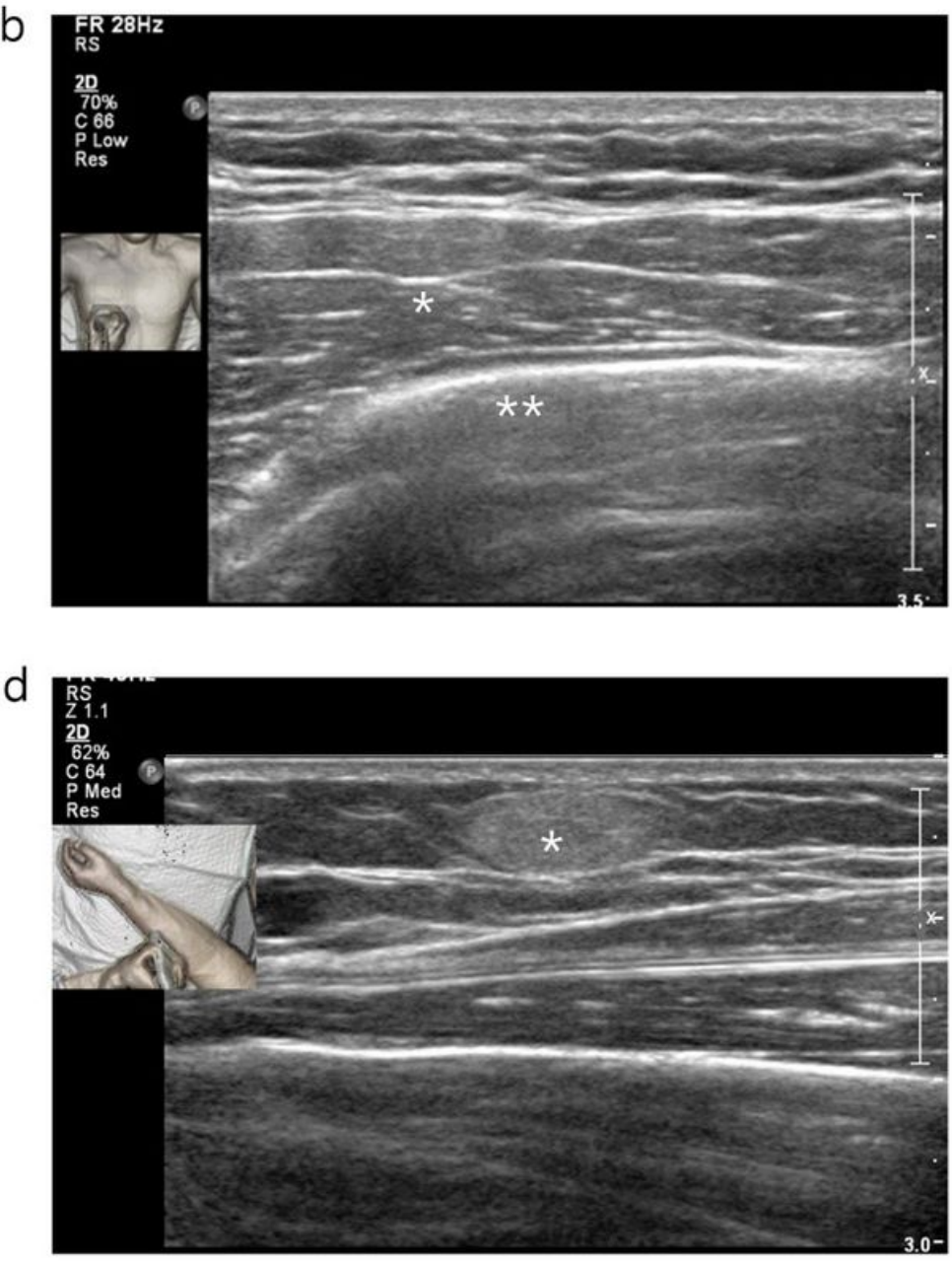

Figure 5

Application of body navigation-loaded ultrasound in various organs of two male participants. (a) An ultrasound image targeted at the right submandibular gland (asterisk). The posture of the participant's neck and location of the transducer are accurately displayed. (b) Ultrasound imaging of the right lateral chest wall allows accurate identification of the pectoralis major muscle (asterisk) and the rib (two asterisks). Without a thumbnail image, other physicians may identify the images to be of muscles and bones, but it would be difficult to determine the exact part of the body of which this image has been taken. (c) The ultrasound image of the left axillary area allows the subcutaneous fat, axillary vessel (asterisk), and pectoralis major muscle (arrow) to be well recognized. (d) An ultrasound image of the subcutaneous nodule (asterisk) of the left arm of another participant. Since the inspection site and transducer information is included, there is no need for text annotation indicating which part of the body the ultrasound image was taken for. 